INPLASY

PROTOCOL

To cite: Zhou et al. Massage for the treatment of knee osteoarthritis: a protocol for an overview of systematic reviews and meta-analysis. Inplasy protocol 202080128. doi: 10.37766/inplasy2020.8.0128

Received: 31 August 2020

Published: 31 August 2020

Corresponding author: Jun Xiong

xiongjun196071@163.com

Author Affiliation:

Jiangxi University of

Traditional Chinese Medicine

Support: 1050Project:

5141900101.

Review Stage at time of this submission: The review has not yet started.

Conflicts of interest:

The authors declare no conflicts of interest.

\section{Massage for the treatment of knee osteoarthritis: a protocol for an overview of systematic reviews and meta-analysis}

Zhou, XC1; Xiong, J2; Chi, ZH³.

Review question / Objective: This overview is an effort to summarize the available evidence from current systematic reviews for the efficacy of massage therapy for knee osteoarthritis.

Condition being studied: Knee osteoarthritis (KOA) is a chronic joint disease that is prone to middle-aged and elderly people.The main clinical manifestations were knee joint pain and joint activity disorder, among which pain was the chief complaint in clinic.

Information sources: Pubmed, Embase, Cochrane Library, Chinese Biomedical Literatures Database(CBM), China National Knowledge Infrastructure (CNKI) , WangFang Database (WF), Chinese Scientific Journal Database (VIP).

INPLASY registration number: This protocol was registered with the International Platform of Registered Systematic Review and Meta-Analysis Protocols (INPLASY) on 31 August 2020 and was last updated on 31 August 2020 (registration number INPLASY202080128).

\title{
INTRODUCTION
}

Review question / Objective: This overview is an effort to summarize the available evidence from current systematic reviews for the efficacy of massage therapy for knee osteoarthritis.
Condition being studied: Knee osteoarthritis (KOA) is a chronic joint disease that is prone to middle-aged and elderly people.The main clinical manifestations were knee joint pain and 
joint activity disorder, among which pain was the chief complaint in clinic.

\section{METHODS}

Participant or population: Knee osteoarthritis patients, regardless of age, gender.

Intervention: The treatment group was mainly massage therapy. The comparison group consisted of those receiving routine care or any intervention other than massage therapy.

Comparator: All cases included in the trial were patients with knee osteoarthritis and met the clinical diagnostic criteria of knee osteoarthritis, without age and race restrictions.

Study designs to be included: All systematic reviews and meta-analysis on the use of massage for knee osteoarthritis.

Eligibility criteria: Published systematic reviews which were reported in Chinese or English, and meet the "PICOS", will be considered for inclusion in this overview.

Information sources: Pubmed, Embase, Cochrane Library, Chinese Biomedical Literatures Database(CBM), China National Knowledge Infrastructure (CNKI), WangFang Database (WF), Chinese Scientific Journal Database (VIP).

Main outcome(s): The Western Ontario and McMaster Universities Osteoarthritis Index scale(WOMAC).

Additional outcome(s): Visual Analogue scale (VAS); Symptom score; Lysholm score; Adverse events.

Data management: (1)We will use NoteExpress and Excel software to extract data. The content will be saved in electronic form. (2)Different review authors will independently screen the titles and abstracts of records obtained by searching the electronic databases to determine potential eligibility. Full texts screening and data extraction will be conducted afterwards independently. Any disagreement regarding study selection will be resolved through discussion or arbitrated by the third author if necessary. In this step, we will use NoteExpress. (3)The research team designed structured data extraction tables, including: the first author, nationality, publication year, patients' basic information, sample size, intervention measures of test group, intervention measures of controlled group, qualitative evaluation method, target outcome (including primary outcome measures and secondary outcome measures), etc. Different review authors will independently extract data. Any disagreement regarding data extraction will be resolved through discussion or arbitrated by the third author if necessary. In this step, we will use Excel.

Quality assessment / Risk of bias analysis: Assessment of Multiple Systematic Reviews 2 (AMSTAR-2) measurement tool,Preferred Reporting Items for Systematic Reviews and Meta-Analyses (PRISMA), Grading of Recommendations Assessment, Development and Evaluation(GRADE) approach.

Strategy of data synthesis: We will provide a narrative description of the findings of the included systematic reviews (SRs). Tables will be produced to detail the included studies and their outcomes. In addition, we will synthesis these reviews and provide pooled treatment effects for all SRs which include the main outcome: the sleep quality values according to the Pittsburgh Sleep Quality Index (PSQI). If necessary, this study will use RevMan5.4 software for data integration and analysis. The measurement data will use the mean difference (MD) as the effect indicator, and the count data will use the odds ratio (OR) as the effect index. Each effect indicator will be given as a point estimate with $95 \%$ confidence interval.The heterogeneity and size of each study result will be judged using statistical methods. For studies with no statistical heterogeneity, the analysis will be performed using a fixed-effect model, whereas a randomized effects 
model will be applied if for studies with significant statistical heterogeneity.

Subgroup analysis: If heterogeneity is evaluated as significant $(12 \geq 50 \%)$ and the trials included are adequate, we will perform a subgroup analysis to explore the potential source of the heterogeneity according to the difference in participant characteristics, interventions, controls, and outcome measures.

Sensibility analysis: According to sample size, methodological quality, and the effect of missing data, sensitivity analysis will be carried out to identify the quality and robustness of the meta-analysis result when the outcome analyses involve a large degree of heterogeneity.

Language: No restriction.

Country(ies) involved: China.

Other relevant information: None.

Keywords: Massage, knee osteoarthritis, protocol, overview.

Contributions of each author:

Author 1 - Xingchen Zhou - Conceive and design this protocol.

Author 2 - Jun Xiong - Revise this protocol; search strategy.

Author 3 - Zhenhai Chi - Data collection; analysis of results. 